\title{
Comparing the Fisher information matrix in record values and random observations for the general class of exponentiated distributions
}

\author{
Fatih Kizılaslan
}

Department of Statistics, Marmara University, Istanbul, Turkey

Received 16 December 2016

Accepted 9 August 2017

\begin{abstract}
In this paper, the Fisher information matrix (FIM) contained in $n$ record values is considered for the two parameter distributions belong to the exponentiated and inverse exponentiated class of distributions. The problem of existence and uniqueness of the maximum likelihood estimates of the parameters for these families are also considered based on record values. The explicit expressions for the elements of the FIM contained in record values as well as in independent and identically (iid) observations are obtained. The Fisher information (FI) matrices are compared by using the relative efficiency, the total information and the total variance. A simulation study is carried out to compare the FI matrices. A real data analysis has also been performed for illustrative purposes.
\end{abstract}

Keywords: Fisher information matrix; record values; exponentiated class of distributions; inverse exponentiated class of distributions.

2000 Mathematics Subject Classification: 62B10, 62F12

\section{Introduction}

Suppose $X$ is absolutely continuous with cumulative density function(cdf) $F(x ; \theta)$ and probability density function (pdf) $f(x ; \theta)$, where $\theta$ is a vector parameter $\left(\theta_{1}, \ldots, \theta_{m}\right)$. Under certain regularity conditions (see, Rao [21]), the FIM, $I(X ; \theta)$, is an $m \times m$ matrix whose $(i, j)$ th element is $I_{i, j}=-E\left(\partial^{2} \ln f(X ; \theta) / \partial \theta_{i} \partial \theta_{j}\right)$. The FI plays an important role in statistical inference through the Cramer-Rao inequality and its association with asymptotic properties of the MLEs. The asymptotic covariance matrix of the MLE of parameters is given by the inverse of the FIM under regularity conditions.

The question "How much information contained in record values?" was addressed by many authors. Comparison of the FI contained in the first $n$ record values with the FI in $n$ iid observations from the same distribution was considered by Ahmadi and Arghani [2]. When the record times were taken into consideration, comparison of these FI was considered by Ahmadi and Arghani [3], Hofmann and Nagaraja [17], Hofmann [15] and Hofmann and Balakrishnan [16]. The FI contained in records, weak records and numbers of records were discussed by Balakrishnan and Stepanov [7]. The FI contained in the first $m$ weak records and the first $m$ (strong) records from a discrete distribution were obtained by Stepanov et al. [23]. In these studies, the FI for only one unknown parameter 
distributions or families were considered based on records and compared with the corresponding FI contained in iid observations. Moreover, when the underlying distribution has an unknown vector parameter, the exact form of the FIM are derived in some cases such as Nagaraja and He [20], Mahmoud and El-Ghafour [19] and Lemonte [18]. However, when the underlying distribution has two or more unknown parameters, the FIM based on record values has not taken into consideration until now.

The aim of this paper is to compare the FI matrices of iid observations and records for the two parameters exponentiated and inverse exponentiated class of distributions. The elements of the FIM based on $n$ record values with $n$ iid observations are obtained analytically. Then, the differences of the elements of the matrices and some relations are derived.

When the interested distribution have a vector parameter $\theta$, the FI matrices are not comparable. In this case, some methods are available to compare the FI of a data set about the unknown parameters and these methods are used greatly in discrimination of the distributions. Recently, there have been many studies concerning the discrimination purposes. Some recent contributions on the topic can be found in the papers by Gupta and Kundu [12,13], Alshunnar et al. [5], Raqab [22] and Ahmad et al. [1]. In these papers, two different measures, the trace of the FIM and the sum of the asymptotic variances of the MLEs of the parameters are generally used to discriminate the interested distributions. In our case, we use these methods to overcome the comparison problem of the FI matrices based on iid data as well as on record data. Moreover, the relative efficiency of iid observations to record data is considered by using the ratio of the determinant of the FI matrices.

The paper is organized as follows. In Section 2, the elements of the FIM for the exponentiated class of distributions are obtained analytically by using both lower record values and iid observations. The existence and uniqueness of the MLEs of the parameters are proved based on lower records. The differences of the FI matrices elements are derived analytically. Then, the relative efficiency, the total information and the total variances of the FI matrices are discussed. In Section 3, the FIM contained in upper record values and iid observations are considered for the inverse exponentiated class of distributions. In Section 4, the obtained results are computed numerically and their results are listed in tables. A real data set analysis is presented. Moreover, the relative efficiency for large values of $\alpha$ is displayed in figures. Finally, we conclude the paper in Section 5.

\section{Fisher information matrix for the exponentiated class of distributions}

The cdf and the pdf of the exponentiated class of distributions are given by

$$
\begin{gathered}
F(x)=\left(1-e^{-\lambda Q(x)}\right)^{\alpha}, x>0, \alpha, \lambda>0, \\
f(x)=\alpha \lambda Q^{\prime}(x) e^{-\lambda Q(x)}\left(1-e^{-\lambda Q(x)}\right)^{\alpha-1}, x>0, \alpha, \lambda>0,
\end{gathered}
$$

where $Q(x)$ is an increasing function with $Q(0)=0$ and $Q(\infty)=\infty$. This family of distributions includes the generalized exponential, generalized Rayleigh (Burr Type X) and generalized Pareto distributions. The existence and uniqueness of the MLEs of the parameters of this family were proved by Ghitany et al. [9] based on complete data. We establish the existence and uniqueness of the MLEs based on lower record values in next. 


\subsection{Fisher information matrix contained in iid observations}

Let $X_{1}, X_{2}, \ldots$ be a sequence of iid continuous random variables from the exponentiated class of distributions with cdf (2.1) and pdf (2.2). Then, the joint density of $X_{1}, \ldots, X_{n}$ is

$$
f\left(x_{1}, \ldots, x_{n} ; \alpha, \lambda\right)=\left(\prod_{i=1}^{n} Q^{\prime}\left(x_{i}\right)\right) \alpha^{n} \lambda^{n} \exp \left\{-\lambda \sum_{i=1}^{n} Q\left(x_{i}\right)+(\alpha-1) \sum_{i=1}^{n} \ln \left(1-e^{-\lambda Q\left(x_{i}\right)}\right)\right\} .
$$

The MLE of $\alpha$ is $\widehat{\alpha}_{X}=-n / \sum_{i=1}^{n} \ln \left(1-e^{-\widehat{\lambda}_{X} Q\left(x_{i}\right)}\right)$ and the MLE of $\lambda$, say $\widehat{\lambda}_{X}$, is a solution of the nonlinear equation

$$
\frac{n}{\lambda}-\sum_{i=1}^{n} \frac{Q\left(x_{i}\right)}{1-e^{-\lambda Q\left(x_{i}\right)}}-\frac{n}{\sum_{i=1}^{n} \ln \left(1-e^{-\hat{\lambda}_{X} Q\left(x_{i}\right)}\right)} \sum_{i=1}^{n} \frac{Q\left(x_{i}\right) e^{-\lambda Q\left(x_{i}\right)}}{1-e^{-\lambda Q\left(x_{i}\right)}}=0 .
$$

The elements of the FIM contained in $n$ iid observations $X_{1}, X_{2}, \ldots, X_{n}$ are obtained by using the formulas 4.253 and 4.261 in Gradshteyn and Ryzhik [11] and are given as

$$
\begin{gathered}
I_{11}^{X}=E\left(-\frac{\partial^{2}}{\partial \alpha^{2}} \ln f\left(x_{1}, \ldots, x_{n} ; \alpha, \lambda\right)\right)=\frac{n}{\alpha^{2}}, \\
I_{12}^{X}=E\left(-\frac{\partial^{2}}{\partial \alpha \partial \lambda} \ln f\left(x_{1}, \ldots, x_{n} ; \alpha, \lambda\right)\right)=-\sum_{i=1}^{n} E\left(\frac{Q\left(x_{i}\right) e^{-\lambda Q\left(x_{i}\right)}}{1-e^{-\lambda Q\left(x_{i}\right)}}\right) \\
=\frac{n \alpha}{\lambda} \int_{0}^{1} t(1-t)^{\alpha-2} \ln t d t \\
=\left\{\begin{array}{cc}
\frac{n \alpha}{\lambda} B(\alpha-1,2)\{\psi(2)-\psi(\alpha+1)\} & , \alpha>1 \\
\frac{n \alpha}{\lambda} \int_{0}^{1} t(1-t)^{\alpha-2} \ln t d t & , 0<\alpha \leq 1
\end{array}\right.
\end{gathered}
$$

and

$$
\begin{aligned}
I_{22}^{X} & =E\left(-\frac{\partial^{2}}{\partial \lambda^{2}} \ln f\left(x_{1}, \ldots, x_{n} ; \alpha, \lambda\right)\right)=\frac{n}{\lambda^{2}}+(\alpha-1) \sum_{i=1}^{n} E\left(\frac{Q^{2}\left(x_{i}\right) e^{-\lambda Q\left(x_{i}\right)}}{\left(1-e^{-\lambda Q\left(x_{i}\right)}\right)^{2}}\right) \\
& =\frac{n}{\lambda^{2}}+\frac{n \alpha(\alpha-1)}{\lambda^{2}} \int_{0}^{1} t(1-t)^{\alpha-3}(\ln t)^{2} d t \\
& =\left\{\begin{array}{cc}
\frac{n}{\lambda^{2}}+\frac{n \alpha(\alpha-1)}{\lambda^{2}} B(\alpha-2,2)\left\{[\psi(2)-\psi(\alpha)]^{2}+\psi^{\prime}(2)-\psi^{\prime}(\alpha)\right\} & , \alpha>2 \\
\frac{n}{\lambda^{2}}+\frac{n \alpha(\alpha-1)}{\lambda^{2}} \int_{0}^{1} t(1-t)^{\alpha-3}(\ln t)^{2} d t & , 0<\alpha \leq 2
\end{array},\right.
\end{aligned}
$$

where $\psi(x)=d \ln \Gamma(x) / d x$ is a Psi function.

It is known that the MLE of $u=u(\theta), \theta=\left(\theta_{1}, \theta_{2}\right)$ is asymptotically normal with mean $u(\theta)$ and asymptotic variance

$$
\operatorname{Var}(\widehat{u})=\sum_{j=1}^{2} \sum_{i=1}^{2} \frac{\partial u}{\partial \theta_{i}} \frac{\partial u}{\partial \theta_{j}} I_{i j}^{-1}
$$

where $I_{i j}^{-1}$ is the $(i, j)$ th element of the inverse of $I(\theta)$, see Rao [21]. Therefore, the asymptotic variance of the MLE of $\alpha$ and $\lambda$ based on iid observations are obtained as

$$
\operatorname{Var}\left(\widehat{\alpha}_{X}\right)=\frac{I_{22}^{X}}{I_{11}^{X} I_{22}^{X}-\left(I_{12}^{X}\right)^{2}}, \operatorname{Var}\left(\widehat{\lambda}_{X}\right)=\frac{I_{11}^{X}}{I_{11}^{X} I_{22}^{X}-\left(I_{12}^{X}\right)^{2}} .
$$

It can be easily seen that $\operatorname{Var}\left(\widehat{\alpha}_{X}\right)$ is independent of $\lambda$. 


\subsection{Fisher information matrix contained in lower record values}

In this subsection, first the existence and uniqueness of the MLEs are proved, and then the elements of the FIM and the asymptotic variance of the MLEs are obtained.

Let $X_{1}, X_{2}, \ldots$ be a sequence of iid continuous random variables with cdf $F(x ; \theta)$ and pdf $f(x ; \theta)$. Let $L_{1}, L_{2}, \ldots$ be the corresponding sequence of lower record values, then the joint density of $L_{1}, \ldots, L_{n}$ is (see, Arnold et al. [6])

$$
f\left(l_{1}, \ldots, l_{n} ; \theta\right)=\prod_{i=1}^{n-1} \frac{f\left(l_{i} ; \theta\right)}{F\left(l_{i} ; \theta\right)} f\left(l_{n} ; \theta\right),-\infty<l_{n}<\ldots<l_{1}<\infty .
$$

Let $X_{1}, X_{2}, \ldots$ be a sequence of iid continuous random variables from the exponentiated class of distributions with cdf (2.1) and pdf (2.2). Then, the joint density of $L_{1}, \ldots, L_{n}$ is given by

$$
\begin{aligned}
f\left(l_{1}, \ldots, l_{n} ; \alpha, \lambda\right)= & \left(\prod_{i=1}^{n} Q^{\prime}\left(l_{i}\right)\right) \alpha^{n} \lambda^{n} \\
& \exp \left\{-\lambda \sum_{i=1}^{n} Q\left(l_{i}\right)-\sum_{i=1}^{n} \ln \left(1-e^{-\lambda Q\left(l_{i}\right)}\right)+\alpha \ln \left(1-e^{-\lambda Q\left(l_{n}\right)}\right)\right\} .
\end{aligned}
$$

The MLE of $\alpha$ is $\widehat{\alpha}_{L}=-n / \ln \left(1-e^{-\widehat{\lambda}_{L} Q\left(l_{n}\right)}\right)$ where $\widehat{\lambda}_{L}$ is a solution of the nonlinear equation

$$
\frac{n}{\lambda}-\sum_{i=1}^{n} \frac{Q\left(l_{i}\right)}{1-e^{-\lambda Q\left(l_{i}\right)}}-\frac{n}{\ln \left(1-e^{-\lambda Q\left(l_{n}\right)}\right)} \frac{Q\left(l_{n}\right) e^{-\lambda Q\left(l_{n}\right)}}{1-e^{-\lambda Q\left(l_{n}\right)}}=0 .
$$

The following theorem shows the existence and uniqueness of the MLEs of $\alpha$ and $\lambda$.

Theorem 2.1. The MLEs of the parameters $\alpha$ and $\lambda$ are unique and given by $\widehat{\alpha}_{L}=-n / \ln (1-$ $\left.e^{-\widehat{\lambda}_{L} Q\left(l_{n}\right)}\right)$ where $\widehat{\lambda}_{L}$ is the solution of the nonlinear equation:

$$
G(\lambda)=\frac{n}{\lambda}-\sum_{i=1}^{n} \frac{Q\left(l_{i}\right)}{1-e^{-\lambda Q\left(l_{i}\right)}}-\frac{n}{\ln \left(1-e^{-\lambda Q\left(l_{n}\right)}\right)} \frac{Q\left(l_{n}\right) e^{-\lambda Q\left(l_{n}\right)}}{1-e^{-\lambda Q\left(l_{n}\right)}} .
$$

Proof It is clear that if the MLE of $\lambda$ is shown to be unique, then the MLE of $\alpha$ will be unique. For this reason, we need to show that the solution of the equation $G(\lambda)=0$ has a unique solution. First, we investigate the limit of $G(\lambda)$ as $\lambda \rightarrow 0$ and $\lambda \rightarrow \infty$. Let $t_{i}=\lambda Q\left(l_{i}\right), i=1, \ldots, m$. Since $Q($.) is an increasing function, $Q(0)=0$ and $Q(\infty)=\infty, Q($.) is a positive function. Then, we have

$$
G(0) \equiv \lim _{\lambda \rightarrow 0} G(\lambda)=\sum_{i=1}^{n} Q\left(l_{i}\right) \lim _{t_{i} \rightarrow 0}\left(\frac{1}{t_{i}}-\frac{1}{1-e^{-t_{i}}}\right)-n Q\left(l_{n}\right) \lim _{t_{n} \rightarrow 0} \frac{e^{-t_{n}} /\left(1-e^{-t_{n}}\right)}{\ln \left(1-e^{-t_{n}}\right)} .
$$

It is easily seen that $G(0)=\infty$ by using the following limits $\lim _{t \rightarrow 0}\left((1 / t)-\left(1 /\left(1-e^{-t}\right)\right)=-1 / 2\right.$ and $\lim _{t \rightarrow 0}\left(e^{-t} /\left(1-e^{-t}\right)\right) / \ln \left(1-e^{-t}\right)=-\infty$. Moreover,

$$
\begin{aligned}
G(\infty) & \equiv \lim _{\lambda \rightarrow \infty} G(\lambda)=-\sum_{i=1}^{n} Q\left(l_{i}\right) \lim _{\lambda \rightarrow \infty}\left(\frac{1}{1-e^{-\lambda Q\left(l_{i}\right)}}\right)-n Q\left(l_{n}\right) \lim _{\lambda \rightarrow \infty} \frac{e^{-\lambda Q\left(l_{n}\right)} /\left(1-e^{-\lambda Q\left(l_{n}\right)}\right)}{\ln \left(1-e^{-\lambda Q\left(l_{n}\right)}\right)} \\
& =-\sum_{i=1}^{n} Q\left(l_{i}\right)+n Q\left(l_{n}\right)=\sum_{i=1}^{n-1}\left(Q\left(l_{n}\right)-Q\left(l_{i}\right)\right)<0,
\end{aligned}
$$

because of $Q\left(l_{i}\right)>Q\left(l_{n}\right) i=1, \ldots, n$ for the lower records $l_{1}>\ldots>l_{i}>\ldots>l_{n}$. 
Hence, we obtain that $\lim _{\lambda \rightarrow 0} G(\lambda)=\infty$ and $\lim _{\lambda \rightarrow \infty} G(\lambda)<0$. By the intermediate value theorem $G(\lambda)$ has at least one root in $(0, \infty)$. If it can be shown that $\partial G(\lambda) / \partial \lambda<0$, then the proof will be completed. The equation (2.8) can be rewritten as follows:

$$
G(\lambda)=G_{1}(\lambda)-n G_{2}(\lambda)
$$

where

$$
\begin{gathered}
G_{1}(\lambda)=\frac{n}{\lambda}-\sum_{i=1}^{n} \frac{Q\left(l_{i}\right)}{1-e^{-\lambda Q\left(l_{i}\right)}}, \\
G_{2}(\lambda)=\frac{G_{3}(\lambda)}{G_{4}(\lambda)}, G_{3}(\lambda)=\frac{Q\left(l_{n}\right) e^{-\lambda Q\left(l_{n}\right)}}{1-e^{-\lambda Q\left(l_{n}\right)}} \text { and } G_{4}(\lambda)=\ln \left(1-e^{-\lambda Q\left(l_{n}\right)}\right) .
\end{gathered}
$$

It is obtained that

$$
G_{1}^{\prime}(\lambda)=\frac{1}{\lambda^{2}} \sum_{i=1}^{n}\left(\frac{Q^{2}\left(l_{i}\right) e^{-\lambda Q\left(l_{i}\right)}}{\left(1-e^{-\lambda Q\left(l_{i}\right)}\right)^{2}}-1\right)=\frac{1}{\lambda^{2}} \sum_{i=1}^{n}\left(\frac{t_{i}^{2} e^{-t_{i}}}{\left(1-e^{-t_{i}}\right)^{2}}-1\right) .
$$

From the Lemma 2 in Ghitany et al. [9], $t^{k} e^{-t}<\left(1-e^{-t}\right)^{k}$ for all $t>0$ and $k=1,2$. When $k=2$, we have $G_{1}^{\prime}(\lambda)<0$ by using this inequality. Moreover,

$$
G_{2}^{\prime}(\lambda)=\left(\frac{G_{3}(\lambda)}{G_{4}(\lambda)}\right)^{2}\left(-e^{-\lambda Q\left(l_{n}\right)} G_{4}(\lambda)-1\right)
$$

It is known that $-\ln (1-x)>x$ for $0<x<1$. By using this inequality for $x=e^{-\lambda Q\left(l_{n}\right)}$, we have $-\ln \left(1-e^{-\lambda Q\left(l_{n}\right)}\right)>e^{-\lambda Q\left(l_{n}\right)}$. Hence, $-e^{\lambda Q\left(l_{n}\right)} G_{4}(\lambda)-1>0$ and $G_{2}^{\prime}(\lambda)>0$. Therefore, it is obtained that $G^{\prime}(\lambda)=G_{1}^{\prime}(\lambda)-n G_{2}^{\prime}(\lambda)<0$.

Finally, we will show that the MLEs of $\alpha$ and $\lambda$ maximizes the log-likelihood function $L(\alpha, \lambda ; \mathbf{l}) \equiv \ln f\left(l_{1}, \ldots, l_{n} ; \alpha, \lambda\right)$. Let $H(\alpha, \lambda)$ be the Hessian matrix of $L(\alpha, \lambda ; \mathbf{l})$ at $(\alpha, \lambda)$. It is clear that

$$
H_{11}\left(\widehat{\alpha}_{L}, \widehat{\lambda}_{L}\right)=-\frac{n}{\widehat{\alpha}^{2}}<0,
$$

and the determinant of the Hessian matrix

$$
\begin{aligned}
D\left(\widehat{\alpha}_{L}, \widehat{\lambda}_{L}\right) & =H_{11}\left(\widehat{\alpha}_{L}, \widehat{\lambda}_{L}\right) H_{22}\left(\widehat{\alpha}_{L}, \widehat{\lambda}_{L}\right)-\left(H_{12}\left(\widehat{\alpha}_{L}, \widehat{\lambda}_{L}\right)\right)^{2} \\
& =-\frac{n}{\widehat{\alpha}^{2}} G_{1}^{\prime}\left(\widehat{\lambda}_{L}\right)-\left(G_{3}^{\prime}\left(\widehat{\lambda}_{L}\right)\right)^{2}\left(e^{-\widehat{\lambda}_{L} Q\left(l_{n}\right)} G_{4}\left(\widehat{\lambda}_{L}\right)+1\right)>0 .
\end{aligned}
$$

Hence, $\left(\widehat{\alpha}_{L}, \widehat{\lambda}_{L}\right)$ is the local maximum of $L(\alpha, \lambda ; \mathbf{l})$. Since there is no singular point of $L(\alpha, \lambda ; \mathbf{l})$ and it has a single critical point then, it is enough to show that the absolute maximum of the function is indeed the local maximum. Assume that there exist a $\widehat{\lambda}_{0}$ in the domain in which $L^{*}\left(\widehat{\lambda}_{0}\right)>L^{*}\left(\widehat{\lambda}_{L}\right)$, where $L^{*}\left(\widehat{\lambda}_{L}\right)=L\left(\widehat{\alpha}_{L}, \widehat{\lambda}_{L} ; \mathbf{l}\right)$. Since $\widehat{\lambda}_{L}$ is the local maximum there should be some point $\lambda_{1}$ in the neighborhood of $\widehat{\lambda}_{L}$ such that $L^{*}\left(\widehat{\lambda}_{L}\right)>L^{*}\left(\lambda_{1}\right)$. Let $k(\lambda)=L^{*}(\lambda)-L^{*}\left(\widehat{\lambda}_{L}\right)$ then $k\left(\widehat{\lambda}_{0}\right)>0, k\left(\lambda_{1}\right)<0$ and $k\left(\widehat{\lambda}_{L}\right)=0$. This implies that $\lambda_{1}$ is a local minimum of the $L^{*}(a)$, but $\widehat{\lambda}_{L}$ is the only critical point so it is a contradiction. Therefore, $\left(\widehat{\alpha}_{L}, \widehat{\lambda}_{L}\right)$ is the absolute maximum of $L(\alpha, \lambda ; \mathbf{l})$. 
Next, we will obtain the elements of the FIM and the asymptotic variances of MLEs. The following results are taken from Corollary 2.1 and Corollary 3.1 in Al-Sirehy and Fisher [4] to obtain the elements of the FIM.

Lemma 2.1. The Beta function $B(\lambda, \mu)$ is usually defined by the integral

$$
B(\lambda, \mu)=\int_{0}^{1} t^{\lambda-1}(1-t)^{\mu-1} d t
$$

for $\lambda, \mu>0$ and more generally, the function $B_{p, q}(\lambda, \mu)$ is defined by the integral

$$
B_{p, q}(\lambda, \mu) \equiv \frac{\partial^{p+q}}{\partial \lambda^{p} \partial \mu^{q}} B(\lambda, \mu)=\int_{0}^{1} t^{\lambda-1}(\ln t)^{p}(1-t)^{\mu-1}(\ln (1-t))^{q} d t,
$$

for $\lambda, \mu>0$ and $p, q=0,1,2, \ldots$. We have the following results for the function $B_{p, q}(\lambda, \mu)$.

(i) For $\lambda \neq 0,-1,-2, \ldots, s=1,2, \ldots$ and $p=0,1,2 \ldots$ we have

$$
B_{p, 1}(\lambda, s)=\sum_{i=0}^{s-1} \sum_{j=1}^{\infty}\left(\begin{array}{c}
s-1 \\
i
\end{array}\right) \frac{(-1)^{i+p+1} p !}{j(\lambda+i+j)^{p+1}} .
$$

(ii) For $\lambda \neq 0,-1,-2, \ldots, s=1,2, \ldots$ and $p=0,1,2 \ldots$ we have

$$
B_{p, 2}(\lambda, s)=\sum_{i=0}^{s-1} \sum_{j=1}^{\infty}\left(\begin{array}{c}
s-1 \\
i
\end{array}\right) \frac{(-1)^{i+p} 2 \phi(j) p !}{(j+1)(\lambda+i+j+1)^{p+1}},
$$

where $\phi(j)=\sum_{k=1}^{j} k^{-1} \equiv \psi(j+1)-\psi(1)$ (see, Gradshteyn and Ryzhik [11]).

First, the FIM contained in a single observation which is the lower record value $L_{i}$ given $L_{i-1}=$ $l_{i-1}$ is considered. It is known that the conditional pdf of $L_{i}$ given $L_{i-1}=l_{i-1}$ is (see, Arnold et al. [6])

$$
f_{L_{i} \mid L_{i-1}}\left(l_{i} \mid L_{i-1}=l_{i-1}\right)=\frac{f\left(l_{i} ; \alpha, \lambda\right)}{F\left(l_{i-1} ; \alpha, \lambda\right)}, l_{i}<l_{i-1}, i=2,3, \ldots
$$

The elements of the FIM can be obtained by using Lemma 2.1 and other expansions. We have

$$
I_{11}^{L}(i)=E\left(-\frac{\partial^{2}}{\partial \alpha^{2}} \ln f_{L_{i} \mid L_{i-1}}\left(l_{i} ; l_{i-1}, \alpha, \lambda\right)\right)=\frac{1}{\alpha^{2}},
$$

for $i=1,2, \ldots, n$ and

$$
\begin{aligned}
I_{12}^{L}(1) & =E\left(-\frac{\partial^{2}}{\partial \alpha \partial \lambda} \ln f\left(l_{1} ; \alpha, \lambda\right)\right)=E\left(-\frac{Q\left(L_{1}\right) e^{-\lambda Q\left(L_{1}\right)}}{1-e^{-\lambda Q\left(L_{1}\right)}}\right) \\
& =\frac{\alpha}{\lambda} \int_{0}^{1} t^{\alpha-2}(1-t) \ln (1-t) d t=\frac{\alpha}{\lambda} B_{0,1}(\alpha-1,2),
\end{aligned}
$$

or $I_{12}^{L}(1)$ can be obtained analytically by using the series expansion of $\ln (1-t)=-\sum_{k=1}^{\infty} t^{k} / k$ as

$$
\begin{aligned}
I_{12}^{L}(1) & =\frac{\alpha}{\lambda} \int_{0}^{1} t^{\alpha-2}(1-t) \ln (1-t) d t=\frac{\alpha}{\lambda} \sum_{k=1}^{\infty}\left(\frac{1}{\alpha+k}-\frac{1}{\alpha+k-1}\right) \\
& =\frac{\alpha}{\lambda}\left(-\frac{1}{\alpha}\right)=-\frac{1}{\lambda} .
\end{aligned}
$$


It is easily seen that the partial sum of the above series converges to $(-1 / \alpha)$.

$$
\begin{aligned}
I_{12}^{L}(i)= & E\left(-\frac{\partial^{2}}{\partial \alpha \partial \lambda} \ln f_{L_{i} \mid L_{i-1}}\left(l_{i} ; l_{i-1}, \alpha, \lambda\right)\right) \\
= & E\left(\frac{Q\left(L_{i-1}\right) e^{-\lambda Q\left(L_{i-1}\right)}}{1-e^{-\lambda Q\left(L_{i-1}\right)}}\right)-E\left(\frac{Q\left(L_{i}\right) e^{-\lambda Q\left(L_{i}\right)}}{1-e^{-\lambda Q\left(L_{i}\right)}}\right) \\
= & -\frac{\alpha^{i-1}(-1)^{i-2}}{\lambda \Gamma(i-1)} \int_{0}^{1} t^{\alpha-2}(1-t)(\ln t)^{i-2} \ln (1-t) d t \\
& +\frac{\alpha^{i}(-1)^{i-1}}{\lambda \Gamma(i)} \int_{0}^{1} t^{\alpha-2}(1-t)(\ln t)^{i-1} \ln (1-t) d t \\
= & -\frac{\alpha^{i-1}(-1)^{i-2}}{\lambda \Gamma(i-1)} B_{i-2,1}(\alpha-1,2)+\frac{\alpha^{i}(-1)^{i-1}}{\lambda \Gamma(i)} B_{i-1,1}(\alpha-1,2),
\end{aligned}
$$

for $i=2,3, . ., n, \alpha \neq 1$, and

$$
\begin{aligned}
I_{22}^{L}(1) & =E\left(-\frac{\partial^{2}}{\partial \lambda^{2}} \ln f\left(l_{1} ; \alpha, \lambda\right)\right)=\frac{1}{\lambda^{2}}+(\alpha-1) E\left(\frac{Q^{2}\left(L_{1}\right) e^{-\lambda Q\left(L_{1}\right)}}{\left(1-e^{-\lambda Q\left(L_{1}\right)}\right)^{2}}\right) \\
& =\frac{1}{\lambda^{2}}+\frac{\alpha(\alpha-1)}{\lambda^{2}} \int_{0}^{1} t^{\alpha-3}(1-t)(\ln (1-t))^{2} d t \\
& =\frac{1}{\lambda^{2}}+\frac{\alpha(\alpha-1)}{\lambda^{2}} B_{0,2}(\alpha-2,2),
\end{aligned}
$$

$$
\begin{aligned}
I_{22}^{L}(i) & =E\left(-\frac{\partial^{2}}{\partial \lambda^{2}} \ln f_{L_{i} \mid L_{i-1}}\left(l_{i} ; l_{i-1}, \alpha, \lambda\right)\right) \\
& =\frac{1}{\lambda^{2}}+(\alpha-1) E\left(\frac{Q^{2}\left(L_{i}\right) e^{-\lambda Q\left(L_{i}\right)}}{\left(1-e^{-\lambda Q\left(L_{i}\right)}\right)^{2}}\right)-\alpha E\left(\frac{Q^{2}\left(L_{i-1}\right) e^{-\lambda Q\left(L_{i-1}\right)}}{\left(1-e^{\left.-\lambda Q\left(L_{i-1}\right)\right)^{2}}\right.}\right) \\
& =\frac{1}{\lambda^{2}}+(\alpha-1) \frac{\alpha^{i}(-1)^{i-1}}{\lambda^{2} \Gamma(i)} \int_{0}^{1} t^{\alpha-3}(1-t)(\ln t)^{i-1}(\ln (1-t))^{2} d t \\
& -\frac{\alpha^{i}(-1)^{i-2}}{\lambda^{2} \Gamma(i-1)} \int_{0}^{1} t^{\alpha-3}(1-t)(\ln t)^{i-2}(\ln (1-t))^{2} d t \\
& =\frac{1}{\lambda^{2}}+(\alpha-1) \frac{\alpha^{i}(-1)^{i-1}}{\lambda^{2} \Gamma(i)} B_{i-1,2}(\alpha-2,2)-\frac{\alpha^{i}(-1)^{i-2}}{\lambda^{2} \Gamma(i-1)} B_{i-2,2}(\alpha-2,2),
\end{aligned}
$$

for $i=2,3, \ldots, n, \alpha \neq 1,2$.

Second, the FIM contained in $n$ lower record values $L_{1}, L_{2}, \ldots, L_{n}$ is considered. The elements of the FIM are obtained as

$$
I_{11}^{L}=E\left(-\frac{\partial^{2}}{\partial \alpha^{2}} \ln f\left(l_{1}, \ldots, l_{n} ; \alpha, \lambda\right)\right)=\frac{n}{\alpha^{2}}
$$




$$
\begin{aligned}
I_{12}^{L} & =E\left(-\frac{\partial^{2}}{\partial \alpha \partial \lambda} \ln f\left(l_{1}, \ldots, l_{n} ; \alpha, \lambda\right)\right)=E\left(-\frac{Q\left(L_{n}\right) e^{-\lambda Q\left(L_{n}\right)}}{1-e^{-\lambda Q\left(L_{n}\right)}}\right) \\
& =\frac{\alpha^{n}(-1)^{n-1}}{\lambda \Gamma(n)} \int_{0}^{1} t^{\alpha-2}(1-t)(\ln t)^{n-1} \ln (1-t) d t \\
& =\frac{\alpha^{n}(-1)^{n-1}}{\lambda \Gamma(n)} B_{n-1,1}(\alpha-1,2),
\end{aligned}
$$

for $n=1,2, \ldots, \alpha \neq 1$, or $I_{12}^{L}$ can be obtained analytically by using the formulas 4.272(6) in Gradshteyn and Ryzhik [11] and the series expansion of $\ln (1-t)=-\sum_{k=1}^{\infty} t^{k} / k$ as

$$
\begin{aligned}
I_{12}^{L} & =\frac{\alpha^{n}}{\lambda \Gamma(n)} \int_{0}^{1} t^{\alpha-2}(1-t)\left(\ln \frac{1}{t}\right)^{n-1} \ln (1-t) d t \\
& =-\frac{\alpha^{n}}{\lambda \Gamma(n)} \sum_{k=1}^{\infty} \frac{1}{k} \int_{0}^{1} t^{\alpha+k-2}(1-t)\left(\ln \frac{1}{t}\right)^{n-1} \\
& =-\frac{\alpha^{n}}{\lambda} \sum_{k=1}^{\infty} \frac{1}{k}\left(\frac{1}{(\alpha+k-1)^{n}}-\frac{1}{(\alpha+k)^{n}}\right) .
\end{aligned}
$$

The partial sum of the above series, $S_{m}$, is

$$
\begin{aligned}
S_{m}= & \sum_{k=1}^{m} \frac{1}{k}\left(\frac{1}{(\alpha+k-1)^{n}}-\frac{1}{(\alpha+k)^{n}}\right) \\
= & \frac{1}{\alpha^{n}}+\frac{1}{(\alpha+1)^{n}}\left(\frac{1}{2}-1\right)+\frac{1}{(\alpha+2)^{n}}\left(\frac{1}{3}-\frac{1}{2}\right)+\frac{1}{(\alpha+3)^{n}}\left(\frac{1}{4}-\frac{1}{3}\right)+ \\
& \ldots+\frac{1}{(\alpha+m-1)^{n}}\left(\frac{1}{m}-\frac{1}{m-1}\right)-\frac{1}{m} \frac{1}{(\alpha+m)^{n}},
\end{aligned}
$$

and, then as $m \rightarrow \infty, S_{m}$ converges to $\left(1 / \alpha^{n}\right)-C^{2}$, where $C^{2}$ is a positive constant depends on $\alpha$ and $n$. Hence,

$$
\begin{gathered}
I_{12}^{L}=-\frac{\alpha^{n}}{\lambda}\left(\frac{1}{\alpha^{n}}-C^{2}\right)=-\frac{1}{\lambda}+\frac{\alpha^{n}}{\lambda} C^{2} . \\
I_{22}^{L}=E\left(-\frac{\partial^{2}}{\partial \lambda^{2}} \ln f\left(l_{1}, \ldots, l_{n} ; \alpha, \lambda\right)\right) \\
=\frac{n}{\lambda^{2}}-\sum_{i=1}^{n} E\left(\frac{Q^{2}\left(L_{i}\right) e^{-\lambda Q\left(L_{i}\right)}}{\left(1-e^{-\lambda Q\left(L_{i}\right)}\right)^{2}}\right)+\alpha E\left(\frac{Q^{2}\left(L_{n}\right) e^{-\lambda Q(L n)}}{\left(1-e^{-\lambda Q\left(L_{n}\right)}\right)^{2}}\right) \\
=\frac{n}{\lambda^{2}}-\sum_{i=1}^{n} \frac{\alpha^{i}(-1)^{i-1}}{\lambda^{2} \Gamma(i)} B_{i-1,2}(\alpha-2,2) \\
+\frac{\alpha^{n+1}(-1)^{n-1}}{\lambda^{2} \Gamma(n)} B_{n-1,2}(\alpha-2,2),
\end{gathered}
$$

for $n=1,2, \ldots$ and $\alpha \neq 1,2 . I_{12}^{L}$ and $I_{22}^{L}$ can be evaluated by using the numerical integral when $\alpha=1,2$. 
Remark 2.1. From the above results the elements of the FIM contained in $n$ lower record values $L_{1}, L_{2}, \ldots, L_{n}$ can be written as

$$
I_{11}^{L}=\sum_{i=1}^{n} I_{11}^{L}(i), I_{12}^{L}=\sum_{i=1}^{n} I_{12}^{L}(i) \text { and } I_{22}^{L}=\sum_{i=1}^{n} I_{22}^{L}(i) .
$$

Hence, the asymptotic variance of $\widehat{\alpha}_{L}$ and $\widehat{\lambda}_{L}$ based on lower record values are obtained as

$$
\operatorname{Var}\left(\widehat{\alpha}_{L}\right)=\frac{I_{22}^{L}}{I_{11}^{L} I_{22}^{L}-\left(I_{12}^{L}\right)^{2}}, \operatorname{Var}\left(\widehat{\lambda}_{L}\right)=\frac{I_{11}^{L}}{I_{11}^{L} I_{22}^{L}-\left(I_{12}^{L}\right)^{2}} .
$$

It can be easily seen that $\operatorname{Var}\left(\widehat{\alpha}_{L}\right)$ is independent of $\lambda$.

\subsection{A comparison of the FIM elements of iid observations and lower records}

In this subsection, first the differences of the FIM elements of iid random sample and lower record values, $I_{12}^{X}-I_{12}^{L}$ and $I_{22}^{X}-I_{22}^{L}$, are obtained analytically. Then, the relative efficiency, the total information and the total variance are discussed to compare the information measures contained in the corresponding FI matrices.

From the equations (2.4), (2.10) and (2.11), we have $I_{12}^{X}=n I_{12}^{L}(1)=-n / \lambda$. Therefore, the difference $I_{12}^{X}-I_{12}^{L}$ is easily obtained by using $I_{12}^{X}$ and the exact form of $I_{12}^{L}$ from equation (2.18)

$$
I_{12}^{X}-I_{12}^{L}=-\frac{(n-1)}{\lambda}-\frac{\alpha^{n}}{\lambda} C^{2}<0 .
$$

From the equations (2.5) and (2.13), we have $I_{22}^{X}=n I_{22}^{L}(1)$. By using the series expansion of $B_{p, q}(\lambda, \mu)$ in Lemma 2.1, we have

$$
\begin{aligned}
I_{22}^{X}-I_{22}^{L} & =\sum_{i=2}^{n} \frac{\alpha(\alpha-1)}{\lambda^{2}}\left[\sum_{j=1}^{\infty} \frac{2 \phi(j)}{j+1}\left(\frac{1}{\alpha+j-1}-\frac{1}{\alpha+j}\right)\right] \\
& -\sum_{i=2}^{n} \frac{\alpha^{i}}{\lambda^{2}}\left[\sum_{j=1}^{\infty} \frac{2 \phi(j)}{j+1}\left(\frac{\alpha-1}{(\alpha+j-1)^{i}}-\frac{\alpha-1}{(\alpha+j)^{i}}-\frac{1}{(\alpha+j-1)^{i-1}}+\frac{1}{(\alpha+j)^{i-1}}\right)\right]
\end{aligned}
$$

The difference in equation (2.22) is investigated for two parts according to $\alpha$.

First, $0<\alpha<1$ case is considered. In this case, since $\alpha>\alpha^{i}, i=2, \ldots, n,(\alpha+j-1)^{i}>(\alpha+$ $j-1), i=2, \ldots, n, j=2,3, \ldots$ and $\phi(1)=1$, we have

$$
\begin{aligned}
I_{22}^{X}-I_{22}^{L} & >\sum_{i=2}^{n} \frac{\alpha^{i}(\alpha-1)}{\lambda^{2}}\left[\sum_{j=1}^{\infty} \frac{2 \phi(j)}{j+1}\left(\frac{1}{\alpha+j-1}-\frac{1}{\alpha+j}-\frac{1}{(\alpha+j-1)^{i}}+\frac{1}{(\alpha+j)^{i}}\right)\right] \\
& -\sum_{i=2}^{n} \frac{\alpha^{i}}{\lambda^{2}}\left[\sum_{j=1}^{\infty} \frac{2 \phi(j)}{j+1}\left(\frac{1}{(\alpha+j)^{i-1}}-\frac{1}{(\alpha+j-1)^{i-1}}\right)\right] \\
& >\sum_{i=2}^{n} \frac{\alpha^{i}(\alpha-1)}{\lambda^{2}} \sum_{j=2}^{\infty} \frac{2 \phi(j)}{j+1}\left(\frac{1}{(\alpha+j)^{i}}-\frac{1}{\alpha+j}\right) \\
& -\sum_{i=2}^{n} \frac{\alpha^{i}}{\lambda^{2}} \sum_{j=2}^{\infty} \frac{2 \phi(j)}{j+1}\left(\frac{1}{(\alpha+j)^{i-1}}-\frac{1}{(\alpha+j-1)^{i-1}}\right) \\
& +\sum_{i=2}^{n}\left[\frac{\alpha^{i}(\alpha-1)}{\lambda^{2}}\left(\frac{1}{\alpha}-\frac{1}{\alpha+1}-\frac{1}{\alpha^{i}}+\frac{1}{(\alpha+1)^{i}}\right)-\frac{\alpha^{i}}{\lambda^{2}}\left(\frac{1}{(\alpha+1)^{i-1}}-\frac{1}{\alpha^{i-1}}\right)\right] .
\end{aligned}
$$


It is clear that $(\alpha+j)^{i-1}>(\alpha+j-1)^{i-1}$ and $(\alpha+j)^{i}>\alpha+j$ for $i=2, \ldots, n, j=1,2,3, \ldots$. Then, the first and the second summations in equation (2.23) are positive. Now, we consider the following series:

$$
D \equiv \sum_{i=2}^{n}\left[\frac{\alpha^{i}(\alpha-1)}{\lambda^{2}}\left(\frac{1}{\alpha}-\frac{1}{\alpha+1}-\frac{1}{\alpha^{i}}+\frac{1}{(\alpha+1)^{i}}\right)-\frac{\alpha^{i}}{\lambda^{2}}\left(\frac{1}{(\alpha+1)^{i-1}}-\frac{1}{\alpha^{i-1}}\right)\right] .
$$

Since, $(\alpha+1)^{i-1}>\alpha+1,(\alpha+1)^{i}>\alpha^{i}$ and $\alpha \geq \alpha^{i-1}$ for $i=2, \ldots, n$, we have

$$
\begin{aligned}
D & >\sum_{i=2}^{n} \frac{\alpha^{i}}{\lambda^{2}}\left[(\alpha-1)\left(\frac{1}{\alpha}-\frac{1}{\alpha+1}-\frac{1}{\alpha^{i}}+\frac{1}{(\alpha+1)^{i}}\right)-\frac{1}{\alpha+1}+\frac{1}{\alpha}\right] \\
& =\sum_{i=2}^{n} \frac{\alpha^{i}}{\lambda^{2}}\left[\frac{1}{\alpha+1}+(\alpha-1)\left(\frac{1}{(\alpha+1)^{i}}-\frac{1}{\alpha^{i}}\right)\right]>0 .
\end{aligned}
$$

Hence, from the equations (2.23) and (2.24), the difference $I_{22}^{X}-I_{22}^{L}>0$.

Second, $\alpha>1$ case is considered. From equation (2.22), we have

$$
\begin{aligned}
I_{22}^{X}-I_{22}^{L}= & \frac{1}{\lambda^{2}} \sum_{i=2}^{n} \sum_{j=1}^{\infty} \frac{2 \phi(j)}{j+1}\left[\frac{\alpha(\alpha-1)}{\alpha+j-1}-\frac{\alpha(\alpha-1)}{\alpha+j}-\frac{\alpha^{i}(\alpha-1)}{(\alpha+j-1)^{i}}+\right. \\
& \left.\frac{\alpha^{i}(\alpha-1)}{(\alpha+j)^{i}}+\frac{\alpha^{i}}{(\alpha+j-1)^{i}}-\frac{\alpha^{i}}{(\alpha+j)^{i-1}}\right] \\
= & \frac{1}{\lambda^{2}} \sum_{i=2}^{n} \sum_{j=1}^{\infty} \frac{2 \phi(j)}{j+1} \frac{f(\alpha)}{(\alpha+j)^{i}(\alpha+j-1)^{i}}
\end{aligned}
$$

where $f(\alpha)=\alpha(\alpha+j)^{i-1}(\alpha+j-1)^{i-1} f_{1}(\alpha)$ and

$$
f_{1}(\alpha)=(\alpha-1)+j(\alpha+j)\left(\frac{\alpha}{\alpha+j-1}\right)^{i-1}-(j+1)(\alpha+j-1)\left(\frac{\alpha}{\alpha+j}\right)^{i-1},
$$

for $i=2, \ldots, n, j=1,2,3, \ldots$. If we can show that $f_{1}(\alpha)>0$ for $\alpha>1$, then $f(\alpha)>0$ and the summation in equation $(2.25)$ will be positive. $f_{1}(\alpha)$ can be rewritten as

$$
f_{1}(\alpha)=j(\alpha+j)\left[\left(\frac{\alpha}{\alpha+j-1}\right)^{i-1}-\left(\frac{\alpha}{\alpha+j}\right)^{i-1}\right]+(\alpha-1)\left[1-\left(\frac{\alpha}{\alpha+j}\right)^{i-1}\right]
$$

Since $\alpha+j>\alpha+j-1$ and $\alpha+j>\alpha$, we can obtain that $f_{1}(\alpha)>0$. Hence, $I_{22}^{X}-I_{22}^{L}>0$ is obtained for $\alpha>1$.

Some comparison methods can be used to compare the FIM contained in $n$ record values with the FIM in $n$ iid observations. We first use the ratio of the determinant of the FI matrices to obtain the relative efficiency of iid data relative to record data. The relative efficiency is defined as

$$
R-e f f=\frac{\operatorname{det}\left(I^{X}\right)}{\operatorname{det}\left(I^{L}\right)}
$$

This measure was also used to compare the FI matrices by different authors such as Barabesi and El-Sharaawi [8] and Hatefi and Jozani [14]. Since the determinants include series expansion, these determinants are not compared analytically. However, the relative efficiency of samples is obtained 
numerically in simulation case and its graphs are displayed in figures. If a value of the relative efficiency is greater than one, it shows that $n$ iid observations provides more information about the parameters $(\alpha, \lambda)$ than record values. In our case, as $I_{22}^{X}-I_{22}^{L}$ and $\left(I_{12}^{L}\right)^{2}-\left(I_{12}^{X}\right)^{2}$ are not comparable in the ratio of the determinants, the obtained numerical results can not be derived analytically. Moreover, it is easily seen that the relative efficiency does not depend on $\lambda$ but depends on $\alpha$. Hence, it is always same for all $\lambda$ when $\alpha$ is fixed.

In the literature, when the fitted distributions are very close to each other, it is very difficult to discriminate the different distributions. Some methods are used for discrimination purposes, one of them to compare the corresponding FI matrices of these distributions based on an interested data set. It is clear that the comparison is not a trivial when the underlying distribution have a vector parameter $\theta$. Two different measures, the total information and the total variance are generally used to compare the FI matrices in the papers Gupta and Kundu [12,13], Alshunnar et al. [5], Raqab [22] and Ahmad et al. [1]. The total information is computed by using the trace of the corresponding FIM and the total variance is the sum of the asymptotic variances of the MLEs of the parameters, i.e. the trace of the inverse of the FIM.

In this paper, we use the aforementioned measures to compare the FIM contained in $n$ record values with the FIM contained in $n$ iid observations. It can be easily seen that the differences of the trace of the FI matrices is positive because

$$
\operatorname{Trace}\left(I^{X}\right)-\operatorname{Trace}\left(I^{L}\right)=\left(I_{11}^{X}-I_{11}^{L}\right)+\left(I_{22}^{X}-I_{22}^{L}\right)=I_{22}^{X}-I_{22}^{L}>0 .
$$

Therefore, the total information of the FIM based on iid observations is always greater than that of record values. This result is observed in Tables 1-2. Moreover, the total variance of the FI matrices are computed numerically in simulation case.

\section{Fisher information matrix for the inverse exponentiated class of distributions}

The inverse exponentiated class of distributions is constructed by using the cdf of $Y$ given in (2.1). When $X=1 / Y$, the survival function of $X$ is given by

$$
S(x)=P(X>x)=\left(1-e^{-\lambda Q(1 / x)}\right)^{\alpha} ; x>0, \alpha, \lambda>0,
$$

and the corresponding pdf of $X$ is

$$
f(x)=\alpha \lambda \frac{Q^{\prime}(1 / x)}{x^{2}} e^{-\lambda Q(1 / x)}\left(1-e^{-\lambda Q(1 / x)}\right)^{\alpha-1} ; x>0, \alpha, \lambda>0 .
$$

This family of distributions includes inverted exponentiated exponential, inverted exponentiated Rayleigh and inverted exponentiated Pareto distributions when $Q(1 / x)=1 / x, Q(1 / x)=1 / x^{2}$ and $Q(1 / x)=\ln (1+1 / x)$, respectively. The existence and uniqueness of the MLEs of the parameters of this family were considered by Ghitany et al. [10] based on complete, progressively Type-I censored and progressively Type-II censored data.

Let $X_{1}, X_{2}, \ldots$ be a sequence of iid continuous random variables from the inverse exponentiated class of distributions with survival function (3.1) and pdf (3.2). In this section, the FIM contained in $n$ iid observation $X_{1}, X_{2}, \ldots, X_{n}$ and $n$ upper record values are considered. 
The joint density of $X_{1}, \ldots, X_{n}$ is

$$
\begin{aligned}
f\left(x_{1}, \ldots, x_{n} ; \alpha, \lambda\right)= & \left(\prod_{i=1}^{n} \frac{Q^{\prime}\left(1 / x_{i}\right)}{x_{i}^{2}}\right) \alpha^{n} \lambda^{n} \\
& \exp \left\{-\lambda \sum_{i=1}^{n} Q\left(1 / x_{i}\right)+(\alpha-1) \sum_{i=1}^{n} \ln \left(1-e^{-\lambda Q\left(1 / x_{i}\right)}\right)\right\} .
\end{aligned}
$$

The elements of the FIM contained in $n$ iid observations $X_{1}, X_{2}, \ldots, X_{n}$ are derived. It is observed that they are the same as in the exponentiated class of distributions case. Therefore, the elements of the FIM and the asymptotic variance of the MLE of $\alpha$ and $\lambda$ are given as in (2.3), (2.4), (2.5) and (2.6), respectively.

Let $U_{1}, U_{2}, \ldots, U_{n}$ be the corresponding sequence of upper record values. Then, the joint density of $U_{1}, U_{2}, \ldots, U_{n}$ is given by

$$
\begin{aligned}
f\left(u_{1}, \ldots, u_{n} ; \alpha, \lambda\right) & =\left(\prod_{i=1}^{n} \frac{Q^{\prime}\left(1 / u_{i}\right)}{u_{i}^{2}}\right) \alpha^{n} \lambda^{n} \\
& \exp \left\{-\lambda \sum_{i=1}^{n} Q\left(1 / u_{i}\right)-\sum_{i=1}^{n} \ln \left(1-e^{-\lambda Q\left(1 / u_{i}\right)}\right)+\alpha \ln \left(1-e^{-\lambda Q\left(1 / u_{n}\right)}\right)\right\},
\end{aligned}
$$

where $u_{1}<\ldots<u_{n}$. In this case, the MLE of $\alpha$ is $\widehat{\alpha}_{U}=-n / \ln \left(1-e^{-\widehat{\lambda}_{U} Q\left(1 / u_{n}\right)}\right)$ where $\widehat{\lambda}_{U}$ is a solution of the nonlinear equation

$$
H(\lambda) \equiv \frac{n}{\lambda}-\sum_{i=1}^{n} \frac{Q\left(1 / u_{i}\right)}{1-e^{-\lambda Q\left(1 / u_{i}\right)}}-\frac{n}{\ln \left(1-e^{-\lambda Q\left(1 / u_{n}\right)}\right)} \frac{Q\left(1 / u_{n}\right) e^{-\lambda Q\left(1 / u_{n}\right)}}{1-e^{-\lambda Q\left(1 / u_{n}\right)}}=0 .
$$

It is easily seen that $Q\left(1 / u_{1}\right)>\ldots>Q\left(1 / u_{i}\right)>\ldots>Q\left(1 / u_{n}\right)$ when $Q($.$) is an increasing function$ and $u_{1}<\ldots<u_{n}$. Since the nonlinear equations given in (2.8) and (3.3) are the same equations, the nonlinear equation $H(\lambda)=0$ has a unique $\widehat{\lambda}_{U}$ solution. Therefore, the MLEs of $\alpha$ and $\lambda$ are unique for the inverse exponentiated class of distributions based on upper record values.

The elements of the FIM contained in $n$ upper record values are obtained and they are the same as in the exponentiated class of distributions case. Thus, the elements of the FIM and the asymptotic variance of the MLE of $\alpha$ and $\lambda$ are given as in (2.15), (2.16), (2.19) and (2.20), respectively.

\section{A comparison study}

In this section, the obtained results in the paper are computed numerically to see which sample have more information than another. The relative efficiency, the total information and the total variances of the FI matrices are computed and their results are listed in Table 1 for different sample sizes $n$ and $(\alpha, \lambda)$ values. The relative efficiency of iid data relative to record data, $R-e f f$, does not depend on $\lambda$ and the graphs of $R-e f f$ versus $\alpha$ are also displayed in Figure 1 for different sample sizes and large values of $\alpha$.

It is observed that the relative efficiency is always greater than one for all cases in Table 1 and Figure 1. However, it can be smaller than one for very large values of $\alpha$. It is observed that the relative efficiency is smaller than one when $\alpha \geq 3142$ and $n=2$. The relative efficiency increases as the sample size $n$ increases and it decreases as $\alpha$ increases when $\lambda$ is fixed. For example, the relative efficiency is greater than one when $\alpha=3142$ and $n=3 . R-e f f>1$ leads to $\operatorname{det}\left(I^{X}\right)>\operatorname{det}\left(I^{L}\right)$ and 

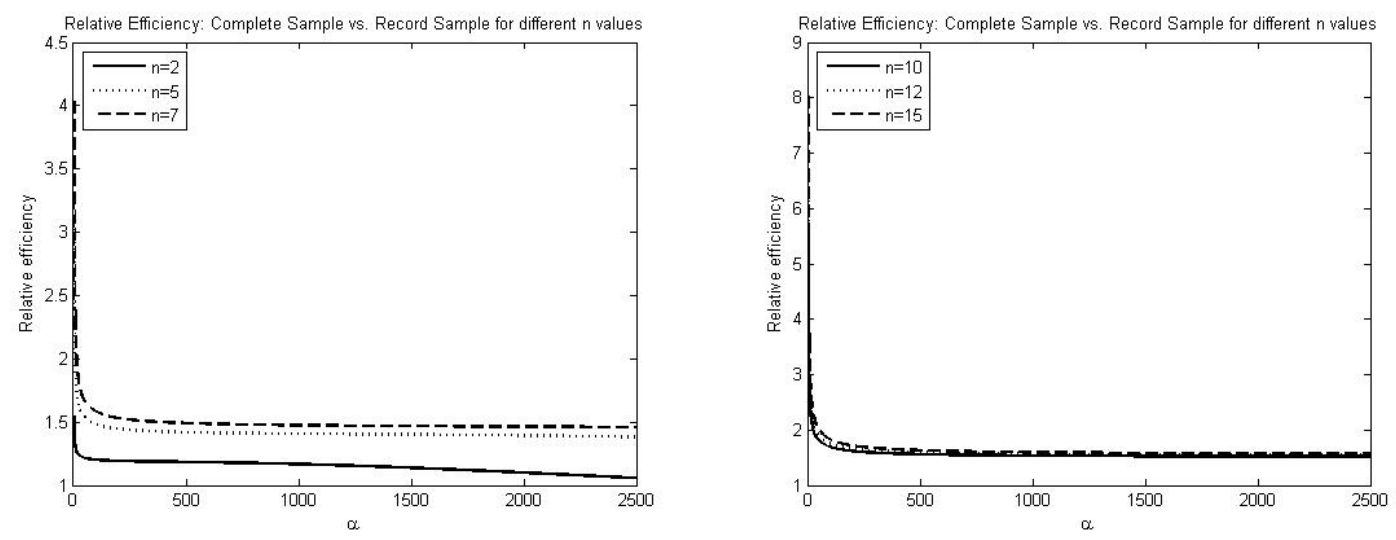

Fig. 1. Relative efficiency versus $\alpha$

then the asymptotic variances of $\widehat{\lambda}, \operatorname{Var}\left(\widehat{\lambda}_{X}\right)$ and $\operatorname{Var}\left(\widehat{\lambda}_{L}\right)$, are always ordered as $\operatorname{Var}\left(\widehat{\lambda}_{X}\right)<\operatorname{Var}\left(\widehat{\lambda}_{L}\right)$. It is also observed that $\operatorname{Var}\left(\widehat{\alpha}_{L}\right)<\operatorname{Var}\left(\widehat{\alpha}_{X}\right)$. Although the asymptotic variances of the MLEs are ordered, the total variances can not be ordered. Since the total informations, Trace $I^{X}$ and Trace $I^{L}$, are ordered as Trace $I^{X}>$ Trace $I^{L}, n$ iid observations have more information than $n$ record values.

Moreover, Monte Carlo simulation is carried out when the underlying distribution is generalized exponential. The MLEs of $\alpha$ and $\lambda$ obtained based on iid data as well as on lower records. Their corresponding FI matrices, relative efficiency, total information and total variances are computed by using these ML estimates and results are listed in Table 2.

From Table 2, the mean square error (MSE) and the variances of the ML estimates decrease as the sample size increases, as expected. We have the following orders: $\operatorname{MSE}\left(\widehat{\alpha}_{X}\right)>\operatorname{MSE}\left(\widehat{\alpha}_{L}\right)$, $\operatorname{MSE}\left(\widehat{\lambda}_{X}\right)<\operatorname{MSE}\left(\widehat{\lambda}_{L}\right), \operatorname{Var}\left(\widehat{\alpha}_{L}\right)<\operatorname{Var}\left(\widehat{\alpha}_{X}\right), \operatorname{Var}\left(\widehat{\lambda}_{X}\right)<\operatorname{Var}\left(\widehat{\lambda}_{L}\right)$. However, the total variance of $I^{X}$ is greater than total variance of $I^{L}$, it is observed in some cases in Table 1.

A real life data set deals with the total seasonal annual rainfall (in inches) recorded at Los Angeles Civic Center from 1994 to 2007 (season 1 July-30 June) is considered for illustrative purposes. This data set can be obtained from the Los Angeles Civic website: htp://www.laalmanac.com/weather/we13.htm. The data are as follows: $(24.35,12.44,12.4,31.01$, $9.09,11.57,17.94,4.42,16.42,9.25,37.96,13.19,3.21)$. We checked the the validity of the generalized exponential distribution based on the parameters $\widehat{\lambda}=0.1166, \widehat{\alpha}=2.9673$, using the Kolmogorov-Smirnov (K-S) test. It is observed that the K-S distance is 0.12916 with a corresponding $p$-value is 0.9625 . Hence, the generalized exponential distribution provides a very good fit to this data set. If only the lower record values of the seasonal rainfall have been observed, these are $\mathbf{r}=(24.35,12.44,12.4,9.09,4.42,3.21)$. To compare the lower records with the same size iid observations, we choose the random observations from the same period as $\mathbf{x}=(31.01,11.57$, $17.94,16.42,9.25,37.96)$. Based on $\mathbf{x}, \widehat{\lambda}=0.1181, \widehat{\alpha}=5.8622$ and its K-S distance and $p$-value are 0.1946 and 0.9438 , respectively. Therefore, the generalized exponential distribution provides a very good fit to $\mathbf{x}$. We compute the MLEs of $(\alpha, \lambda)$, an asymptotic variances and total informations based on lower record values as well as iid observations. These results are listed in Table 3.

From Table 3, it is observed that $\operatorname{Var}\left(\widehat{\alpha}_{L}\right)<\operatorname{Var}\left(\widehat{\alpha}_{X}\right), \operatorname{Var}\left(\widehat{\lambda}_{X}\right)<\operatorname{Var}\left(\widehat{\lambda}_{L}\right)$, the total variance and the total information of data $\mathbf{x}$ are greater than that of data $\mathbf{r}$. These results are similar to those found in the Tables 1 and 2. 
Table 1. Relative efficiency, differences of some elements and trace of the FI matrices and total variances for different values of $\alpha$ and $\lambda$.

\begin{tabular}{|c|c|c|c|c|c|c|c|c|c|}
\hline \multicolumn{10}{|c|}{$\alpha=0.5, \lambda=5$} \\
\hline$n$ & $R-e f f$ & Trace for $I^{X}$ & Trace for $I^{L}$ & Total Var. $I^{X}$ & Total Var. $I^{L}$ & $\operatorname{Var}\left(\widehat{\alpha}_{X}\right)$ & $\operatorname{Var}\left(\widehat{\alpha}_{L}\right)$ & $\operatorname{Var}\left(\lambda_{X}\right)$ & $\operatorname{Var}\left(\lambda_{L}\right)$ \\
\hline 2 & 1.6702 & 8.0425 & 8.0227 & 32.8450 & 54.7218 & 0.1738 & 0.1548 & 32.6713 & 54.5670 \\
\hline 3 & 2.3344 & 12.0638 & 12.0229 & 21.8967 & 50.9427 & 0.1158 & 0.0969 & 21.7808 & 50.8458 \\
\hline 4 & 2.9970 & 16.0851 & 16.0229 & 16.4225 & 49.0282 & 0.0869 & 0.0701 & 16.3356 & 48.9581 \\
\hline 5 & 1.9132 & 20.1064 & 20.0229 & 13.1380 & 47.8875 & 0.0695 & 0.0548 & 13.0685 & 47.8328 \\
\hline 8 & 5.6553 & 32.1702 & 32.0229 & 8.2113 & 46.2248 & 0.0434 & 0.0331 & 8.1678 & 46.1918 \\
\hline 10 & 6.9885 & 40.2127 & 40.0229 & 6.5690 & 45.6909 & 0.0348 & 0.0261 & 6.5343 & 45.6648 \\
\hline 12 & 8.3229 & 48.2553 & 48.0229 & 5.4742 & 45.3415 & 0.0290 & 0.0216 & 5.4452 & 45.3199 \\
\hline 15 & 10.3256 & 60.3191 & 60.0229 & 4.3793 & 44.9973 & 0.0232 & 0.0172 & 4.3562 & 44.9802 \\
\hline 20 & 13.6651 & 80.4255 & 80.0229 & 3.2845 & 44.6582 & 0.0174 & 0.0128 & 3.2671 & 44.6455 \\
\hline 25 & 17.0054 & 100.5318 & 100.0229 & 2.6276 & 44.4572 & 0.0139 & 0.0102 & 2.6137 & 44.4470 \\
\hline 50 & 33.7111 & 201.0636 & 200.0229 & 1.3138 & 44.0604 & 0.0070 & 0.0050 & 1.3069 & 44.0554 \\
\hline \multicolumn{10}{|c|}{$\alpha=1.5, \lambda=5$} \\
\hline 2 & 1.4801 & 1.0026 & 0.9543 & 19.7348 & 27.8016 & 2.2390 & 1.9061 & 17.4958 & 25.8955 \\
\hline 3 & 1.9178 & 1.5040 & 1.4009 & 13.1565 & 23.5037 & 1.4927 & 1.1344 & 11.6639 & 22.3693 \\
\hline 4 & 2.3365 & 2.0053 & 1.8461 & 9.8674 & 21.2250 & 1.1195 & 0.7853 & 8.7479 & 20.4397 \\
\hline 5 & 2.7469 & 2.5066 & 2.2908 & 7.8939 & 19.8170 & 0.8956 & 0.5930 & 6.9983 & 19.2240 \\
\hline 8 & 3.9672 & 4.0106 & 3.6242 & 4.9337 & 17.6876 & 0.5598 & 0.3352 & 4.3740 & 17.3254 \\
\hline 10 & 4.7830 & 5.0132 & 4.5131 & 3.9470 & 16.9953 & 0.4478 & 0.2587 & 3.4992 & 16.7366 \\
\hline 12 & 5.6025 & 6.0159 & 5.4020 & 3.2891 & 16.5470 & 0.3732 & 0.2104 & 2.9160 & 16.3366 \\
\hline 15 & 6.8370 & 7.5198 & 6.7354 & 2.6313 & 16.1136 & 0.2985 & 0.1643 & 2.3328 & 15.9492 \\
\hline 20 & 8.9034 & 10.0264 & 8.9576 & 1.9735 & 15.6976 & 0.2239 & 0.1204 & 1.7496 & 15.5772 \\
\hline 25 & 10.9754 & 12.5330 & 11.1798 & 1.5788 & 15.4569 & 0.1791 & 0.0950 & 1.3997 & 15.3619 \\
\hline 50 & 21.3602 & 25.0661 & 22.2909 & 0.7894 & 14.9948 & 0.0896 & 0.0462 & 0.6998 & 14.9486 \\
\hline \multicolumn{10}{|c|}{$\alpha=2, \lambda=5$} \\
\hline 2 & 1.4384 & 0.6447 & 0.5853 & 19.9405 & 26.0415 & 4.4746 & 3.7950 & 15.4659 & 22.2465 \\
\hline 3 & 1.8262 & 0.9670 & 0.8392 & 13.2937 & 21.0674 & 2.9830 & 2.2384 & 10.3106 & 18.8290 \\
\hline 4 & 2.1898 & 1.2893 & 1.0906 & 9.9702 & 18.4676 & 2.2373 & 1.5337 & 7.7330 & 16.9339 \\
\hline 5 & 2.5418 & 1.6116 & 1.3411 & 7.9762 & 16.8708 & 1.7898 & 1.1465 & 6.1864 & 15.7243 \\
\hline 8 & 3.5748 & 2.5786 & 2.0915 & 4.9851 & 14.4545 & 1.1186 & 0.6327 & 3.8665 & 13.8219 \\
\hline 10 & 4.2614 & 3.2233 & 2.5916 & 3.9881 & 13.6641 & 0.8949 & 0.4829 & 3.0932 & 13.1812 \\
\hline 12 & 4.9507 & 3.8679 & 3.0916 & 3.3234 & 13.1508 & 0.7458 & 0.3896 & 2.5777 & 12.7612 \\
\hline 15 & 5.9904 & 4.8349 & 3.8416 & 2.6587 & 12.6547 & 0.5966 & 0.3017 & 2.0621 & 12.3530 \\
\hline 20 & 7.7345 & 6.4466 & 5.0916 & 1.9940 & 12.1813 & 0.4475 & 0.2191 & 1.5466 & 11.9621 \\
\hline 25 & 9.4868 & 8.0582 & 6.3416 & 1.5952 & 11.9098 & 0.3580 & 0.1720 & 1.2373 & 11.7378 \\
\hline 50 & 18.2868 & 16.1165 & 12.5916 & 0.7976 & 11.3958 & 0.1790 & 0.0829 & 0.6186 & 11.3129 \\
\hline \multicolumn{10}{|c|}{$\alpha=5, \lambda=5$} \\
\hline 2 & 1.3354 & 0.3730 & 0.2678 & 53.5282 & 51.3337 & 42.0465 & 36.0012 & 11.4817 & 15.3325 \\
\hline 3 & 1.2663 & 0.5594 & 0.3258 & 35.6855 & 33.3258 & 28.0310 & 21.0512 & 7.6545 & 12.2746 \\
\hline 4 & 1.8375 & 0.7459 & 0.3750 & 26.7641 & 24.7272 & 21.0233 & 14.1781 & 5.7409 & 10.5491 \\
\hline 5 & 2.0521 & 0.9324 & 0.4202 & 21.4113 & 19.8039 & 16.8186 & 10.3790 & 4.5927 & 9.4249 \\
\hline 8 & 2.6404 & 1.4919 & 0.5465 & 13.3821 & 12.9442 & 10.5116 & 5.3650 & 2.8704 & 7.5792 \\
\hline 10 & 3.0121 & 1.8648 & 0.6278 & 10.7056 & 10.8568 & 8.4093 & 3.9400 & 2.2963 & 6.9168 \\
\hline 12 & 3.3776 & 2.2378 & 0.7084 & 8.9214 & 9.5397 & 7.0078 & 3.0762 & 1.9136 & 6.4635 \\
\hline 15 & 3.9222 & 2.7972 & 0.8288 & 7.1371 & 8.2942 & 5.6062 & 2.2897 & 1.5309 & 6.0045 \\
\hline 20 & 4.8313 & 3.7296 & 1.0290 & 5.3528 & 7.1348 & 4.2047 & 1.5876 & 1.1482 & 5.5472 \\
\hline 25 & 5.7480 & 4.6620 & 1.2290 & 4.2823 & 6.4887 & 3.3637 & 1.2090 & 0.9185 & 5.2798 \\
\hline 50 & 10.4186 & 9.3241 & 2.2290 & 2.1411 & 5.3328 & 1.6819 & 0.5478 & 0.4593 & 4.7849 \\
\hline \multicolumn{10}{|c|}{$\alpha=10, \lambda=5$} \\
\hline 2 & 1.2827 & 0.4885 & 0.3392 & 241.8476 & 215.4032 & 231.9457 & 202.7018 & 9.9019 & 12.7014 \\
\hline 3 & 1.4931 & 0.7327 & 0.3938 & 161.2317 & 129.3803 & 154.6305 & 119.5240 & 6.6012 & 9.8563 \\
\hline 4 & 1.6667 & 0.9770 & 0.4309 & 120.9238 & 88.8891 & 115.9729 & 80.6372 & 4.9509 & 8.2519 \\
\hline 5 & 1.8192 & 1.2212 & 0.4587 & 96.7390 & 66.0951 & 92.7783 & 58.8898 & 3.9607 & 7.2054 \\
\hline 8 & 2.2122 & 1.9540 & 0.5161 & 60.4619 & 35.3262 & 57.9864 & 29.8501 & 2.4755 & 5.4761 \\
\hline 10 & 2.4469 & 2.4424 & 0.5445 & 48.3695 & 26.3853 & 46.3891 & 21.5395 & 1.9804 & 4.8458 \\
\hline 12 & 2.6708 & 2.9309 & 0.5694 & 40.3079 & 20.9164 & 38.6576 & 16.5086 & 1.6503 & 4.4077 \\
\hline 15 & 2.9952 & 3.6637 & 0.6035 & 32.2463 & 15.9109 & 30.9261 & 11.9564 & 1.3202 & 3.9545 \\
\hline 20 & 3.5213 & 4.8849 & 0.6564 & 24.1848 & 11.4432 & 23.1946 & 7.9565 & 0.9902 & 3.4868 \\
\hline 25 & 4.0412 & 6.1061 & 0.7074 & 19.3478 & 9.0580 & 18.5557 & 5.8567 & 0.7921 & 3.2013 \\
\hline 50 & 6.6678 & 12.2122 & 0.9580 & 9.6739 & 5.0599 & 9.2778 & 2.4189 & 0.3961 & 2.6410 \\
\hline \multicolumn{10}{|c|}{$\alpha=20, \lambda=5$} \\
\hline 2 & 1.2461 & 0.7147 & 0.5152 & 1281.3 & 1150.8 & 1272.369 & 1139.675 & 8.9636 & 11.1695 \\
\hline 3 & 1.4176 & 1.0721 & 0.6111 & 854.2217 & 690.2013 & 848.2460 & 681.7304 & 5.9757 & 8.4710 \\
\hline 4 & 1.5518 & 1.4295 & 0.6771 & 640.6663 & 470.8775 & 636.1845 & 463.9227 & 4.4818 & 6.9548 \\
\hline 5 & 1.6647 & 1.7869 & 0.7256 & 512.5330 & 346.4738 & 508.9476 & 340.5052 & 3.5854 & 5.9686 \\
\hline 8 & 1.9382 & 2.8590 & 0.8168 & 320.3332 & 177.3846 & 318.0923 & 173.0412 & 2.2409 & 4.3434 \\
\hline 10 & 2.0925 & 3.5737 & 0.8537 & 256.2665 & 128.1020 & 254.4738 & 124.3508 & 1.7927 & 3.7512 \\
\hline 12 & 2.2348 & 4.2885 & 0.8807 & 213.5554 & 98.0126 & 212.0615 & 94.6739 & 1.4939 & 3.3387 \\
\hline 15 & 2.4346 & 5.3606 & 0.9100 & 170.8443 & 70.6073 & 169.6492 & 67.6976 & 1.1951 & 2.9097 \\
\hline 20 & 2.7466 & 7.1475 & 0.9427 & 128.1333 & 46.4150 & 127.2369 & 43.9531 & 0.8964 & 2.4619 \\
\hline 25 & 3.0444 & 8.9343 & 0.9654 & 102.5066 & 33.7225 & 101.7895 & 31.5393 & 0.7171 & 2.1831 \\
\hline 50 & 4.4753 & 17.8686 & 1.0400 & 51.2533 & 13.3501 & 50.8948 & 11.7455 & 0.3585 & 1.6046 \\
\hline
\end{tabular}


Table 2. Estimates and some results about the FIM for the generalized exponential distribution

\begin{tabular}{|c|c|c|c|c|c|c|c|c|c|c|c|c|c|}
\hline \multirow[b]{2}{*}{$n$} & \multirow[b]{2}{*}{$R-e f f$} & \multicolumn{6}{|c|}{ based on iid data for $(\alpha, \lambda)=(6,2)$} & \multicolumn{6}{|c|}{ based on record data for $(\alpha, \lambda)=(6,2)$} \\
\hline & & $\widehat{\alpha}$ & $\widehat{\lambda}$ & Trace for $I^{X}$ & Total Var. $I^{X}$ & $\operatorname{Var}\left(\widehat{\alpha}_{X}\right)$ & $\operatorname{Var}\left(\widehat{\lambda}_{X}\right)$ & $\widehat{\alpha}$ & $\widehat{\lambda}$ & Trace for $I^{L}$ & Total Var. $I^{L}$ & $\operatorname{Var}\left(\widehat{\alpha}_{L}\right)$ & $\operatorname{Var}\left(\hat{\lambda}_{L}\right)$ \\
\hline 5 & & $5.42 \times 10^{6}$ & 2.6692 & & & & & 8652.4 & 3.7282 & & & & \\
\hline 7 & & 16499 & 1.4449 & & & & & 297.1895 & 3.0447 & & & & \\
\hline \multirow[t]{2}{*}{10} & 34.1752 & 14.5437 & 2.3710 & 11.0717 & 5453.4 & 5452.9 & 0.4886 & 8.4581 & 2.7598 & 2.1128 & 32.8367 & 30.8593 & 1.9774 \\
\hline & & 2150.3 & 0.7589 & & & & & 38.4226 & 2.4702 & & & & \\
\hline \multicolumn{2}{|l|}{$n$} & \multicolumn{6}{|c|}{ based on iid data for $(\alpha, \lambda)=(5,3)$} & \multicolumn{6}{|c|}{ based on record data for $(\alpha, \lambda)=(5,3)$} \\
\hline \multirow[t]{2}{*}{5} & 731.0519 & 32.8464 & 4.0819 & 2.4441 & $3.249 \times 10^{5}$ & $3.249 \times 10^{5}$ & 2.9876 & 12.4133 & 4.3280 & 0.9313 & 1732.8 & 1726 & 6.7812 \\
\hline & & $4.18 \times 10^{4}$ & 4.3088 & & & & & 601.821 & 6.2136 & & & & \\
\hline \multirow[t]{2}{*}{7} & 57.0307 & 17.5345 & 3.8258 & 3.2930 & 16755 & 16753 & 1.8804 & 8.0860 & 4.0563 & 1.0070 & 84.3860 & 79.0660 & 5.3200 \\
\hline & & 4363.6 & 2.9178 & & & & & 68.1722 & 4.8722 & & & & \\
\hline \multirow[t]{2}{*}{10} & 19.2888 & 10.7101 & 3.5650 & 4.6175 & 525.7142 & 524.5639 & 1.1503 & 6.6838 & 3.9370 & 1.1411 & 18.8519 & 14.3822 & 4.4698 \\
\hline & & 345.596 & 1.7715 & & & & & 18.1956 & 4.3703 & & & & \\
\hline
\end{tabular}

Table 3. Results for the real life example

\begin{tabular}{ccccccc}
\hline data & $\widehat{\alpha}$ & $\widehat{\lambda}$ & Trace for $I$ & Total Var. $I$ & $\operatorname{Var}(\widehat{\alpha})$ & $\operatorname{Var}(\widehat{\lambda})$ \\
\hline $\mathbf{r}$ & 4.4778 & 0.0946 & 562.1825 & 6.1319 & 6.1287 & 0.0033 \\
$\mathbf{x}$ & 5.8622 & 0.1181 & 1763.1 & 20.7401 & 20.7381 & 0.0021 \\
\hline
\end{tabular}

Since the results for the exponentiated class of distributions and the inverse exponentiated class of distributions are common, all numerical results obtained for the exponentiated class of distributions are also valid for the inverse exponentiated class of distributions.

\section{Conclusions}

In this paper, we have derived explicit expressions of the FIM for the two parameter exponentiated class of distributions based on record values as well as on iid observations. We have obtained some relations between the FIM contained in record values and the FIM contained iid observations. It is obtained that the total information based on iid observations is greater than that of record values. From the numerical results, it is observed that using the record values instead of the same number iid observations reduces the asymptotic variance of one parameter and increases the asymptotic variance of another parameter. A real life data is also presented to illustrate obtained results in the paper.

\section{References}

[1] Ahmad, M.A., Raqab, M.Z., Kundu, D., Discriminating between the generalized Rayleigh and Weibull distributions: Some comparative studies, Communications in Statistics-Simulation and Computation (2016) DOI:10.1080/03610918.2015.1136415.

[2] Ahmadi, J., Arghami, N.R., On the Fisher information in record values, Metrika 53 (2001) 195-206.

[3] Ahmadi, J., Arghami, N.R., Comparing the Fisher information in record values and IID observations, Statistics 37 (2003) 435-441.

[4] Al-Sirehy, F., Fisher, B., Results on the beta function and the incomplete beta function, International Journal of Applied Mathematics 26 (2013) 191-201.

[5] Alshunnar, F.S., Raqab, M.Z., Kundu, D., On the comparison of the Fisher information of the lognormal and Weibull distributions, Journal of Applied Statistics 37 (2010) 391-404. 
[6] Arnold, B.C., Balakrishnan, N., Nagaraja, H.N., Records, (Wiley, New York, 1998).

[7] Balakrishnan, N., Stepanov, A., On the Fisher information in record data, Statistics \& Probability Letters 76 (2006) 537-545.

[8] Barabesi, L., El-Sharaawi, A., The efficiency of ranked set sampling for parameter estimation, Statistics \& Probability Letters 53 (2001) 189-199.

[9] Ghitany, M.E., Al-Jarallah, R.A., Balakrishnan, N., On the existence and uniqueness of the MLEs of the parameters of a general class of exponentiated distributions, Statistics 47 (2013) 605-612.

[10] Ghitany, M.E., Tuan, V.K., Balakrishnan, N., Likelihood estimation for a general class of inverse exponentiated distributions based on complete and progressively censored data, Journal of Statistical Computation and Simulation 84 (2014) 96-106.

[11] Gradshteyn, I.S., Ryzhik, I.M., Table of Integrals, Series and Products, (seventh ed. Academic Press, Boston, 1994).

[12] Gupta, R.D., Kundu, D., Discriminating between Weibull and generalized exponential distributions, Computational Statistics \& Data Analysis 43, (2003) 179-196.

[13] Gupta, R.D., Kundu, D., On the comparison of Fisher information of the Weibull and GE distributions, Journal of Statistical Planning and Inference 136 (2006) 3130-3144.

[14] Hatefi, A., Jozani, J.M., Fisher information in different types of perfect and imperfect ranked set samples from finite mixture models, Journal of Multivariate Analysis 119 (2013) 16-31.

[15] Hofmann, G., Comparing the Fisher information in record data and random observations, Statistical Papers 45 (2004) 517-528.

[16] Hofmann, G., Balakrishnan, N., Fisher information in $k$-records, Annals of the Institute of Statistical Mathematics 56 (2004) 383-396.

[17] Hofmann, G., Nagaraja, H.N., Fisher information in record data, Metrika 57 (2003) 177-193.

[18] Lemonte, A. J., A note on the Fisher information matrix of the Birnbaum-Saunders distribution, Journal of Statistical Theory and Applications 15(2) (2016) 196-205.

[19] Mahmoud, M.R., El-Ghafour, A.S.A., Fisher information matrix for the generalized Feller-Pareto distribution, Communications in Statistics-Theory and Methods 44 (2015) 4396-4407.

[20] Nagaraja, H.N., He, Q., Fisher information in censored samples from the Marshall-Olkin bivariate exponential distribution, Communications in Statistics-Theory and Methods 44 (2015) 4172-4184.

[21] Rao, C.R., Linear Statistical Inference and Its Applications, (second ed. John Wiley \& Sons, New York, 1973).

[22] Raqab, M.Z., Discriminating between the generalized Rayleigh and Weibull distributions, Journal of Applied Statistics 40 (2013) 1480-1493.

[23] Stepanov, A.V., Balakrishnan, N., Hofmann, G., Exact distribution and Fisher information of weak record values, Statistics \& Probability Letters 64 (2003) 69-81. 